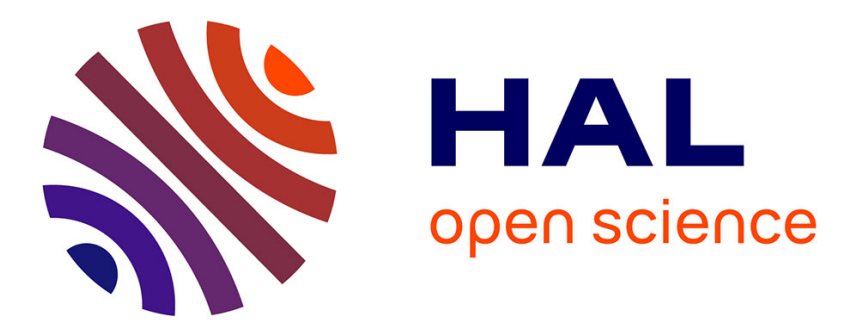

\title{
Noise characteristics of a non-degenerate Optical Parametric Oscillator - Application to quantum noise reduction
}

\author{
C. Fabre, E. Giacobino, A. Heidmann, S. Reynaud
}

\section{- To cite this version:}

C. Fabre, E. Giacobino, A. Heidmann, S. Reynaud. Noise characteristics of a non-degenerate Optical Parametric Oscillator - Application to quantum noise reduction. Journal de Physique, 1989, 50 (10), pp.1209-1225. 10.1051/jphys:0198900500100120900 . jpa-00210990

\section{HAL Id: jpa-00210990 https://hal.science/jpa-00210990}

Submitted on 1 Jan 1989

HAL is a multi-disciplinary open access archive for the deposit and dissemination of scientific research documents, whether they are published or not. The documents may come from teaching and research institutions in France or abroad, or from public or private research centers.
L'archive ouverte pluridisciplinaire HAL, est destinée au dépôt et à la diffusion de documents scientifiques de niveau recherche, publiés ou non, émanant des établissements d'enseignement et de recherche français ou étrangers, des laboratoires publics ou privés. 
Classification

$42.50 \mathrm{Dr}-42.50 \mathrm{~Kb}-42.65 \mathrm{Ky}$

\title{
Noise characteristics of a non-degenerate Optical Parametric Oscillator - Application to quantum noise reduction
}

\author{
C. Fabre, E. Giacobino, A. Heidmann and S. Reynaud \\ Laboratoire de Spectroscopie Hertzienne de l'Ecole Normale Supérieure (*) Université P. et \\ M. Curie 75252 PARIS Cédex 05, France
}

(Reçu le 7 novembre 1988, accepté le 13 janvier 1989)

\begin{abstract}
Résumé. - On présente une analyse semi-classique des caractéristiques de bruit d'un oscillateur paramétrique optique non dégénéré opérant au-dessus du seuil, permettant d'obtenir l'expression des fluctuations quantiques des intensités et des phases des champs émis. On met l'accent plus particulièrement sur l'étude du bruit existant sur la différence des intensités entre les deux champs produits. On étudie l'effet sur cette quantité des pertes, du déséquilibre, des désaccords et de l'excès de bruit de pompe. On trouve une réduction significative du bruit quantique dans un large domaine de l'espace des paramètres.

Abstract. - A semi-classical analysis of the noise characteristics of a non degenerate, above threshold, optical parametric oscillator is presented, yielding expressions for the quantum fluctuations of the output field intensities and phases. Particular emphasis is given to the study of the noise on the intensity difference between the two generated fields. The effect of losses, unbalance, detunings and excess pump noise is investigated. A significant quantum noise reduction is found over a broad domain of the parameter space.
\end{abstract}

\section{Introduction.}

Among the processes which generate light with «non-classical» properties, the optical parametric interaction turns out to be a prototypic one [1-6]. The process involves the generation of two « signal » fields at frequencies $\omega_{1}$ and $\omega_{2}$ by a nonlinear crystal irradiated with a pump field at frequency $\omega_{0}$ (energy conservation requires $\omega_{0}=\omega_{1}+\omega_{2}$ ). The crystal can be placed in a resonant optical cavity operating either below or above oscillation threshold depending on the pump power.

Up to now, most of the work have concentrated on Optical Parametric Amplifiers (OPA) (below threshold operation), which have been shown both theoretically [7-11] and experimentally [12] to yield squeezed states of light.

(*) Laboratoire associé au C.N.R.S., à l'Ecole Normale Supérieure et à l'Université Pierre et Mạie Curie. 
The same device, operating above threshold (Optical Parametric Oscillator: OPO) also yields non-classical states of light. In particular, we have predicted [13,14] that, in the nondegenerate case, the OPO produces intense «twin-beams » with highly correlated intensity fluctuations. As a result, the fluctuations on the difference between the intensities of the two beams are reduced with respect to the standard quantum limit $[15,16]$. We have experimentally shown evidence of such a noise reduction effect yielding $30 \%$ of « intensity squeezing » [17]. This result has been recently bettered to about $70 \%$ of intensity noise reduction [18].

The noise reduction on the intensity difference can be understood very simply by the following argument: in parametric down conversion, the nonlinear crystal emits pairs of simultaneous photons at frequencies $\omega_{1}$ and $\omega_{2}$ each time a pump photon is annihilated, generating « twin » beams which have exactly the same photon statistics : there is no noise on the intensity difference. In the OPO, the cavity placed around the crystal induces some decorrelation between the two beams : the numbers of photons delivered in the two output beams are expected to be nearly equal only when counted during a time long compared to the cavity storage time. In other words, measuring the noise spectrum $S_{I}(\omega)$ of the difference $I$ between the signal beam intensities must reveal a reduction of photon noise inside the cavity bandwidth. This simple argument can be developed on quantum mechanical grounds [19] to yield the expression of $S_{I}(\omega)$.

The purpose of the present paper is to give a comprehensive theoretical overview of the noise properties of the various observable quantities in the OPO, using another approach [14]. Such results are very useful for a better insight into the OPO properties, but also to optimize the various parameters in order to get a maximum noise reduction. The first three sections of this paper give the outline of the method in the resonant case (no detuning for the pump and signal fields). Section 4 is devoted to the discussion of the various noise spectra in the simplest case of resonance and balance (equal loss parameters for the two signal fields). In section 5 , we focus on the intensity difference noise spectrum in the general case of unbalance and detuning. The last section shows the effect of an external balancing on this noise spectrum.

\section{Basic equations.}

The fluctuations characteristics of the OPO emission can be computed very simply using the following «semi-classical» approach [14]. The dynamics of small field fluctuations are described by linearizing the classical equations of motion in the vicinity of the stationary state. We consider that these field fluctuations are driven by the vacuum fluctuations entering the cavity through the coupling mirror. It can be shown [20] that standard quantum methods [16] lead to the same results.

The signal fields are confined in an optical ring cavity (Fig. 1); the two fields can be separated from each other by either their frequencies $\omega_{1}$ and $\omega_{2}$ (with $\omega_{1}$ close to $\left.\omega_{2}\right)$ or their polarizations, which is the experimental case of references $[17,18]$. The pump field at frequency $\omega_{0}$ is also confined in the cavity, and we allow the transmission coefficients of the coupling mirror to be different from one another for the three fields. Other internal losses in the cavity are also accounted for. Perfect phase matching is assumed. If the one-pass gain and losses are small, the equations of motion for the classical amplitudes $\alpha_{1}, \alpha_{2}, \alpha_{0}$ associated with the annihilation operators $a_{1}, a_{2}, a_{0}$ can be written as differential equations :

$$
\begin{aligned}
& \tau \dot{\alpha}_{1}+\left(\gamma_{1}+\mu_{1}\right) \alpha_{1}=2 \chi \alpha_{0} \alpha_{2}^{*}+\sqrt{2 \gamma_{1}} \alpha_{1}^{\text {in }}+\sqrt{2 \mu_{1}} \beta_{1}^{\text {in }} \\
& \tau \dot{\alpha}_{2}+\left(\gamma_{2}+\mu_{2}\right) \alpha_{2}=2 \chi \alpha_{0} \alpha_{1}^{*}+\sqrt{2 \gamma_{2}} \alpha_{2}^{\text {in }}+\sqrt{2 \mu_{2}} \beta_{2}^{\text {in }} \\
& \tau \dot{\alpha}_{0}+\left(\gamma_{0}+\mu_{0}\right) \alpha_{0}=-2 \chi \alpha_{1} \alpha_{2}+\sqrt{2 \gamma_{0}} \alpha_{0}^{\text {in }}+\sqrt{2 \mu_{0}} \beta_{0}^{\text {in }}
\end{aligned}
$$




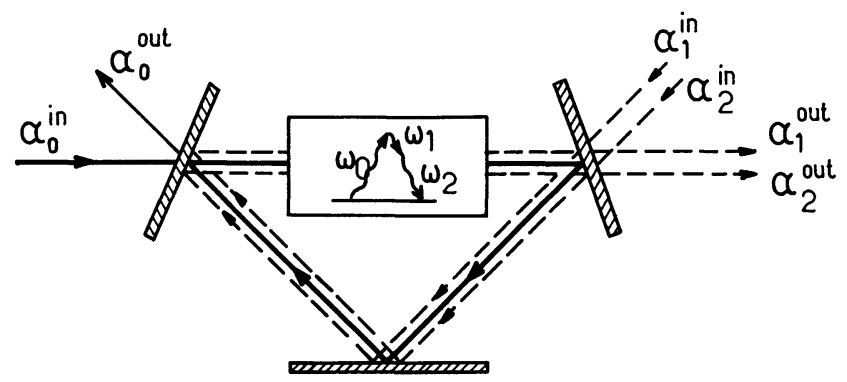

Fig. 1. - Scheme of an Optical Parametric Oscillator. $\alpha_{0}^{\text {in }}, \alpha_{1}^{\text {in }}, \alpha_{2}^{\text {in }}$ are the incoming pump and signal fieds. $\alpha_{0}^{\text {out }}, \alpha_{1}^{\text {out }}, \alpha_{2}^{\text {out }}$ the outgoing corresponding fields.

The cavity round-trip time $\tau$ is assumed to be the same for all three fields. The one pass loss parameters $\gamma_{i}$ and $\mu_{i}(i=0,1,2)$ are associated respectively with the output mirror and with all other loss mechanisms (the $\mu_{i}$ are due to crystal absorption, surface scattering, imperfections of other mirrors...). The $\gamma_{i}$ are related to the amplitude reflection coefficients $r_{i}$ and the amplitude transmission coefficients $t_{i}$ through :

$$
\left\{\begin{array}{l}
r_{i}=1-\gamma_{i} \\
t_{i}=\sqrt{2 \gamma_{i}}
\end{array}\right.
$$

We will denote $\gamma_{i}^{\prime}$ the total loss coefficient $\gamma_{i}+\mu_{i}$. Equations (1) are written at exact resonance for the three fields with the cavity modes. The non-resonant case will be considered in section 5 .

The quantities $\alpha_{i}^{\text {in }}$ and $\beta_{i}^{\text {in }}(i=0,1,2)$ are the incoming fields, associated with the coupling mirror and with the internal loss mechanism respectively. In the following, only the incoming pump field $\alpha_{0}^{\text {in }}$ has a non-zero mean value.

From the values of the fields $\alpha_{1}$ inside the cavity, we will then deduce the outgoing fields $\alpha_{i}^{\text {out }}$ by the following equations relating the fields on the output mirror :

$$
\alpha_{i}^{\text {out }}=t_{i} \alpha_{i}-\alpha_{i}^{\text {in }}
$$

\section{Stationary values.}

The stationary mean field solutions $\bar{\alpha}_{i}$ are calculated by taking $\bar{\alpha}_{i}^{\text {in }}=\bar{\beta}_{i}^{\text {in }}=0(i=1,2)$. A choice of phases is made where $\bar{\alpha}_{0}, \bar{\alpha}_{1}$ and $\bar{\alpha}_{2}$ are real. Actually, whereas the phase of either $\bar{\alpha}_{0}$ or $\bar{\alpha}_{0}^{\text {in }}$ can be arbitrarily chosen, the equations do not fix the value of the phase difference between $\bar{\alpha}_{1}$ and $\bar{\alpha}_{2}$. We will come back (in Sect. 4.2 ) to the phase diffusion process associated with this feature.

There is a non-trivial solution to equations (1), given by :

$$
\begin{aligned}
\gamma_{i}^{\prime} \bar{\alpha}_{i}^{2} & =\frac{\gamma_{0}^{\prime} \gamma_{1}^{\prime} \gamma_{2}^{\prime}}{4 \chi^{2}}(\sigma-1) \quad(i=1,2) \\
\bar{\alpha}_{0}^{2} & =\frac{\gamma_{1}^{\prime} \gamma_{2}^{\prime}}{4 \chi^{2}}
\end{aligned}
$$


when the pump parameter $\sigma$ is larger than 1 (i.e. above threshold) :

$$
\sigma=2 \sqrt{\frac{2 \chi^{2} \gamma_{0}}{\gamma_{0}^{\prime 2} \gamma_{1}^{\prime} \gamma_{2}^{\prime}}} \alpha_{0}^{\text {in }}
$$

The pump parameter can also be written in terms of the pump intensity threshold $P_{0}$ and the incident pump intensity $P$ :

$$
\begin{aligned}
& \sigma=\sqrt{P / P_{0}} \\
& P_{0}=\gamma_{0}^{\prime 2} \gamma_{1}^{\prime} \gamma_{2}^{\prime} / 8 \chi^{2} \gamma_{0}
\end{aligned}
$$

Let us note that the outgoing signal intensities obey a simple relation :

$$
\bar{I}_{1}^{\text {out }} / \bar{I}_{2}^{\text {out }}=\gamma_{1} \gamma_{2}^{\prime} / \gamma_{2} \gamma_{1}^{\prime}
$$

with $\bar{I}_{i}^{\text {out }}=2 \gamma_{i} \bar{\alpha}_{i}^{2}(i=1,2)$. They are equal in the absence of internal losses $\left(\mu_{i}=0\right.$; $\gamma_{i}^{\prime}=\gamma_{i}$ ) even if the mirror transmissions $\gamma_{1}$ and $\gamma_{2}$ are different. This is not surprising, since all the «twin » photons produced by the parametric splitting have to leave the cavity, for any value of the transmission coefficients.

\section{Derivation of the noise spectra.}

In order to obtain the fluctuation dynamics, we linearize the evolution equations around the mean values given by equation (4). Setting $\alpha_{i}=\bar{\alpha}_{i}+\delta \alpha_{i}$ and using equations (1), we obtain :

$$
\begin{gathered}
\tau \delta \dot{\alpha}_{1}+\gamma_{1}^{\prime} \delta \alpha_{1}=\sqrt{\gamma_{1}^{\prime} \gamma_{2}^{\prime} \delta \alpha_{2}^{*}}+\sqrt{\gamma_{0}^{\prime} \gamma_{1}^{\prime}(\sigma-1)} \delta \alpha_{0} \\
+\sqrt{2 \gamma_{1}} \delta \alpha_{1}^{\text {in }}+\sqrt{2 \mu_{1}} \delta \beta_{1}^{\text {in }} \\
\tau \delta \dot{\alpha}_{2}+\gamma_{2}^{\prime} \delta \alpha_{2}=\sqrt{\gamma_{2}^{\prime} \gamma_{1}^{\prime} \delta \alpha_{1}^{*}}+\sqrt{\gamma_{0}^{\prime} \gamma_{2}^{\prime}(\sigma-1)} \delta \alpha_{0} \\
+\sqrt{2 \gamma_{2}} \delta \alpha_{2}^{\text {in }}+\sqrt{2 \mu_{2}} \delta \beta_{2}^{\text {in }} \\
\tau \delta \dot{\alpha}_{0}+\gamma_{0}^{\prime} \delta \alpha_{0}=-\sqrt{\gamma_{0}^{\prime} \gamma_{2}^{\prime}(\sigma-1)} \delta \alpha_{2}-\sqrt{\left.\gamma_{0}^{\prime} \gamma_{1}^{\prime}(\sigma-1)\right)} \delta \alpha_{1} \\
+\sqrt{2 \gamma_{0}} \delta \alpha_{0}^{\text {in }}+\sqrt{2 \mu_{0}} \delta \beta_{0}^{\text {in }}
\end{gathered}
$$

Introducing the real and imaginary parts of the fields, we set :

$$
\left\{\begin{array}{l}
p_{i}=\delta \alpha_{i}+\delta \alpha_{i}^{*} \\
q_{i}=-i\left(\delta \alpha_{i}-\delta \alpha_{i}^{*}\right)
\end{array}(i=0,1,2)\right.
$$

with similar notations for the input and output fields.

As we have chosen the stationary solutions to be real, $p_{i}$ gives the amplitude fluctuations, while $q_{i}$ is proportional to the phase fluctuations. The differential equations (8) are then transformed into algebraic equations for the Fourier components $\tilde{p}_{i}(\omega), \tilde{q}_{i}(\omega)$ relating the inner fluctuations to the input ones. The normalization factor for the input fluctuations is chosen so that :

$$
V_{p_{i}}^{\text {in }}(\omega)=\left\langle\left|\tilde{p}_{i}^{\text {in }}(\omega)\right|^{2}\right\rangle=1
$$


Then one deduces from equation (3) the expressions of the output fluctuations. The squeezing spectrum $S_{p_{i}}(\omega)$ for the parameter $p_{i}$ is directly given by the output fluctuation variance :

$$
S_{p_{i}}(\omega)=V_{i}^{\text {out }}(\omega)=\left\langle\left|p_{i}^{\text {out }}(\omega)\right|^{2}\right\rangle
$$

By a similar procedure, one obtains the fluctuation spectra of the $q_{i}$ 's outside the OPO cavity.

\section{Fluctuation spectra in the balanced case.}

As a first result, we will give the various fluctuation spectra in the balanced case, when the losses and transmission factors are the same for the two generated field. We will call $\gamma^{\prime}, \gamma$ and $\mu$ the common value of $\gamma_{i}^{\prime}, \gamma_{i}$ and $\mu_{i}(i=1,2)$ respectively.

4.1 INTENSITY DIFFERENCE. - Since the mean intensities of the twin beams are equal, the fluctuations in the intensity difference $I=I_{1}-I_{2}$ are proportional to the fluctuations of the amplitude difference. Let us denote :

$$
r=\frac{1}{\sqrt{2}}\left(p_{1}-p_{2}\right)
$$

(with similar notations for the input and output fields). From equations (8) we obtain the following equation for $r$ :

$$
\tau \dot{r}+2 \gamma^{\prime} r=\sqrt{2 \gamma} r^{\text {in }}+\sqrt{2 \mu} r^{\text {in }}
$$

The important point to notice is that $r$ is insensitive to the pump fluctuations, which cancel in the difference. We thus obtain for the Fourier component $\tilde{r}(\omega)$ of $r$ :

$$
\tilde{r}(\omega)=\frac{1}{2 \gamma^{\prime}+i \omega \tau}\left[\sqrt{2 \gamma} \tilde{r}^{\text {in }}(\omega)+\sqrt{2 \mu} \tilde{r}^{\text {in }}(\omega)\right]
$$

The outgoing field $r^{\text {out }}$ is easily derived from (3) :

$$
r^{\text {out }}=\sqrt{2 \gamma} r-r^{\text {in }}
$$

Hence combining equations (14) and (15) leads to :

$$
\tilde{r}^{\text {out }}(\omega)=\frac{2 \gamma-2 \gamma^{\prime}-i \omega \tau}{2 \gamma^{\prime}+i \omega \tau} \tilde{r}^{\text {in }}(\omega)+\frac{2 \sqrt{\gamma \mu}}{2 \gamma^{\prime}+i \omega \tau} \tilde{r}^{\text {in }}(\omega)
$$

$\tilde{r}^{\text {in }}$ and $\tilde{r}^{i n}$ are independent random variables, with variances normalized to 1 . The squeezing spectrum for the $r$ component is given by :

$$
\begin{aligned}
S_{r}(\omega) & =\left|\frac{2 \mu+i \omega \tau}{2 \gamma^{\prime}+i \omega \tau}\right|^{2}+\left|\frac{2 \sqrt{\gamma \mu}}{2 \gamma^{\prime}+i \omega \tau}\right|^{2} \\
& =\frac{4 \mu \gamma^{\prime}+\omega^{2} \tau^{2}}{4 \gamma^{\prime 2}+\omega^{2} \tau^{2}}
\end{aligned}
$$

In the balanced case, the intensity noise spectrum $S_{I}(\omega)$ is thus proportional to the amplitude noise spectrum :

$$
S_{I}(\omega)=S_{0} \frac{4 \mu \gamma^{\prime}+\omega^{2} \tau^{2}}{4 \gamma^{\prime 2}+\omega^{2} \tau^{2}}
$$




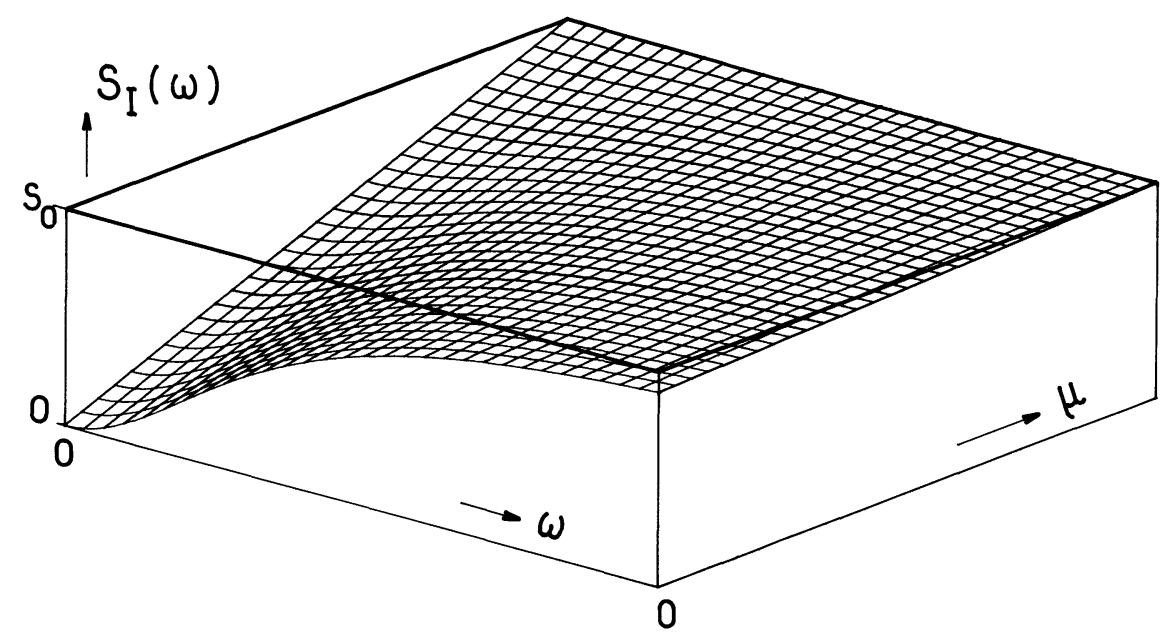

Fig. 2. - Noise spectrum $S_{I}(\omega)$ on the intensity difference as a function of the losses $\mu$. The cavity bandwidth $\gamma^{\prime}$ (transmission $\gamma+$ losses $\mu$ ) is kept constant. $S_{0}$ is the standard quantum noise level on a beam of intensity $I_{1}+I_{2}\left(0<\omega \tau<6 \gamma^{\prime}, 0<\mu<\gamma^{\prime}\right)$.

where $S_{0}$ is the shot noise on a beam with a total intensity equal to the sum of the intensities of the twin beams. $S_{I}(\omega)$ is displayed as a function of the frequency $\omega$ and the losses $\mu$ in figure (2) for a constant value of the cavity bandwidth $\gamma^{\prime}$. The shot noise is reduced for any value of these parameters. The noise reduction is significant only inside the cavity bandwidth $\left(\omega \tau<2 \gamma^{\prime}\right)$. When there is no internal loss, the noise is completely suppressed at zero frequency.

In presence of losses, the minimum noise is $\mu / \gamma^{\prime}=\mu /(\mu+\gamma)$. This factor is the proportion of emitted photons which do not reach the photodetector. This implies that the extra losses associated with $\mu$ have to be kept much smaller than losses through the output mirror. Let us emphasize that the noise spectrum is independent of the pump parameters (mean intensity and fluctuations).

4.2 Phase Difference. - The fluctuation spectrum of the phase difference between the twin fields can be calculated in the same way. Setting :

$$
s=\frac{1}{\sqrt{2}}\left(q_{1}-q_{2}\right)
$$

one gets :

$$
S_{s}(\omega)=1+\frac{4 \gamma \gamma^{\prime}}{\omega^{2} \tau^{2}}
$$

This expression diverges when $\omega$ goes to 0 , which is characteristic of a phase diffusion process. One can deduce the fluctuation spectrum $S_{\Omega}(\omega)$ of the beat frequency between the two signal modes :

$$
\Omega=\omega_{1}-\omega_{2}=\frac{1}{\sqrt{2} \bar{\alpha}_{1}} \dot{s}
$$


(where $\bar{\alpha}_{1}=\bar{\alpha}_{2}$ is the mean signal field). One gets :

$$
\begin{aligned}
S_{\Omega}(\omega) & =\frac{1}{2\left(\bar{\alpha}_{1}^{\text {out }}\right)^{2}} \omega^{2} S_{s}(\omega) \\
& =\frac{1}{2 I_{1}^{\text {out }}}\left(\frac{4 \gamma \gamma^{\prime}}{\tau^{2}}+\omega^{2}\right)
\end{aligned}
$$

The noise spectrum $S_{\Omega}(\omega)$ of the beat frequency between the two signal fields can be divided into two distinct parts : in the low frequency regime, $S_{\Omega}(\omega)$ is a constant, which corresponds to the Schawlow-Townes phase diffusion process occurring on the phase difference [16, 22]. In the high-frequency regime, $S_{\Omega}(\omega)$ is proportional to $\omega^{2}$, which describes the effect of the vacuum fluctuations reflected on the output mirror [23].

4.3 PHASE AND INTENSITY SUMS. - In contrast with the difference variables $r$ and $s$, the fluctuations of the phase sum $q=\left(q_{1}+q_{2}\right) / \sqrt{2}$ and of the intensity sum $p=\left(p_{1}+p_{2}\right) / \sqrt{2}$ are sensitive to the pump fluctuations $\delta \alpha_{0}$.

In order to simplify the calculations, from now on, we will restrict ourselves to the following case : the cavity finesse for the pump is much lower than for the signals $\left(\gamma_{0}^{\prime} \gg \gamma^{\prime}\right)$, allowing the adiabatic elimination procedure; for noise frequencies small compared to $\gamma_{0}^{\prime} / \tau$, we neglect $i \omega \tau$ in the Fourier transform of equation (8c), yielding :

$$
\gamma_{0}^{\prime} \delta \alpha_{0}=-\sqrt{\gamma_{0}^{\prime} \gamma^{\prime}(\sigma-1)}\left(\delta \alpha_{1}+\delta \alpha_{2}\right)+\sqrt{2 \gamma_{0}} \delta \alpha_{0}^{\text {in }}+\sqrt{2 \mu_{0}} \delta \beta_{0}^{\text {in }}
$$

This value for $\delta \alpha_{0}$ is then inserted in equations ( $8 \mathrm{a}$ ) and (8b). We obtain dynamical equations for $\delta \alpha_{1}$ and $\delta \alpha_{2}$ containing the effect of pump fluctuations. Using the same method as before, one gets for the noise spectra of $q$ and $p$ :

$$
\begin{gathered}
S_{q}(\omega)=1-\frac{4 \gamma \gamma^{\prime}}{\omega^{2} \tau^{2}+4 \gamma^{\prime 2} \sigma^{2}} \\
S_{p}(\omega)=1+\frac{4 \gamma \gamma^{\prime}}{\omega^{2} \tau^{2}+4 \gamma^{\prime 2}(\sigma-1)^{2}}
\end{gathered}
$$

These quantities now depend on $\sigma$, that is on the pump power.

In figure (3), the noise spectrum for the sum of the phases, $S_{q}$ is displayed as a function of $\omega$ and $\sigma$. Complete noise suppression is obtained at zero frequency when $\mu=0$ and at threshold $(\sigma=1)$. This result is the equivalent in the non-degenerate OPO of the squeezing observed in the degenerate case on the phase component [12].

The noise spectrum $S_{I_{1}+I_{2}}$ for the sum of intensities is equal to :

$$
S_{I_{1}+I_{2}}(\omega)=S_{0} S_{p}(\omega)
$$

( $S_{0}$ is the same shot noise as in Eq. (18)). At zero frequency, $S_{I_{1}+I_{2}}$ diverges close to threshold $(\sigma \rightarrow 1)$ and goes down to the shot noise limit when $\sigma \rightarrow \infty$. This component exhibits amplified fluctuations, in the same way as the amplitude component in the degenerate case [12].

4.4 INTENSITY FLUCTUATIONS ON A SINGLE BEAM. - An interesting feature appears on the fluctuation spectrum of a single beam intensity $S_{I_{1}}(\omega)$. This quantity is given by the following expression :

$$
S_{I_{1}}(\omega)=\frac{S_{0}}{2}\left(1-\frac{8 \gamma \gamma^{\prime 3} \sigma(\sigma-2)}{\left(\omega^{2} \tau^{2}+4 \gamma^{\prime 2}\right)\left(\omega^{2} \tau^{2}+4 \gamma^{\prime 2}(\sigma-1)^{2}\right)}\right)
$$




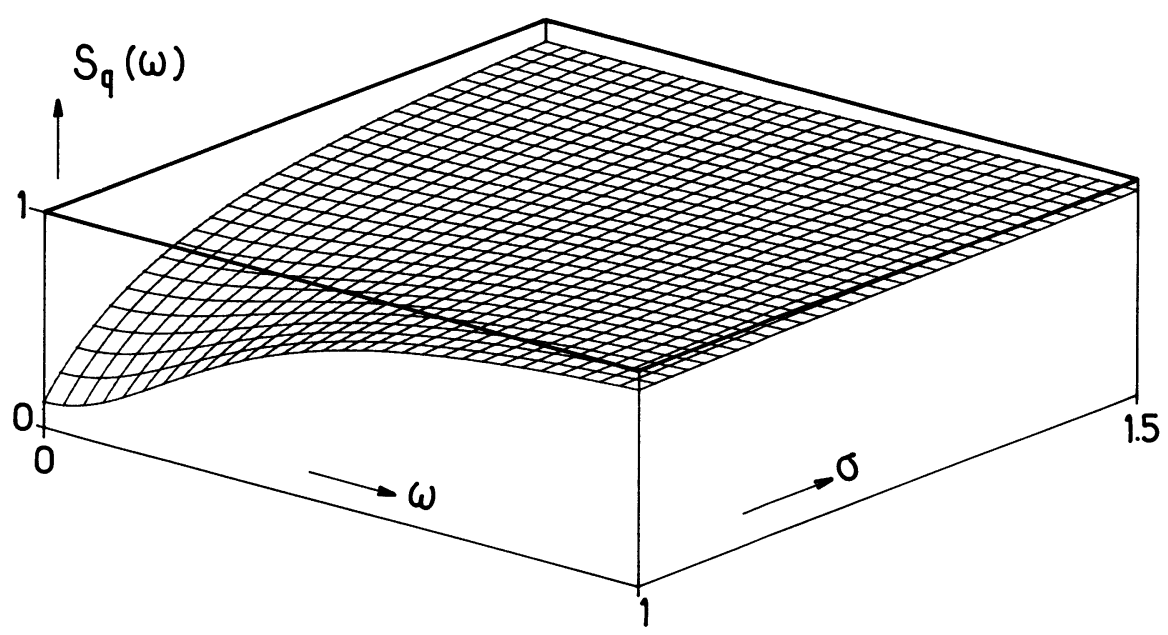

Fig. 3. - Noise spectrum $S_{q}(\omega)$ on the phase quadrature sum $q$ as a function of the pump amplitude parameter $\sigma=\sqrt{P / P_{0}} . S_{q}(\omega)=1$ corresponds to the standard quantum noise on this quantity. $\sigma=1$ corresponds to the oscillation threshold. $\left(\mu=0.1 \gamma^{\prime}, 0<\omega \tau<6 \gamma^{\prime}, 1<\sigma<1.5\right)$.

where $\frac{S_{0}}{2}$ is the shot noise on one of the twin beams. Starting from above shot noise near threshold, $S_{I_{1}}(\omega)$ goes below the shot noise for $\sigma>2$ in a bandwidth of the order of the cavity bandwidth (Fig. 4). At best, for $\omega=0$, and $\sigma \rightarrow \infty$ :

$$
S_{I_{1}}(0)=\frac{S_{0}}{2}\left(1-\frac{\gamma}{2 \gamma^{\prime}}\right)
$$

If there are no internal losses, the shot noise is reduced by a factor 2 at very high pump power values [15].

4.5 EFFECT OF DETUNING ON THE SPECTRUM OF THE INTENSITY DIFFERENCE. - It is well known that, in a doubly resonant OPO, the four oscillation conditions (energy conservation, phase matching condition, cavity resonance conditions for both signal and idler) are only fulfilled for a discrete series of cavity length values. In practice, oscillation occurs only in very small length intervals around these values. This is the cause of the well-known high sensitivity of the OPO to vibrations [21]. Thus, the OPO length has to be actively stabilized so that it delivers a constant output intensity. In the experiment of reference [17], the OPO length is stabilized on the edge of one of such small oscillation domains, leading to a slight mismatch between the OPO frequencies and the cavity eigenfrequencies.

As a consequence, our previous calculation for the intensity difference fluctuation spectrum does not correspond to the experimental conditions. Let us assume that the detunings $\varphi_{1}$ and $\varphi_{2}$ between the round trip phases of the signal modes and the cavity resonances are small. Under these conditions, equations (1) for the evolution of $\alpha_{1}$ and $\alpha_{2}$ are replaced by :

$$
\left\{\begin{array}{l}
\tau \dot{\alpha}_{1}+\left(\gamma^{\prime}+i \varphi_{1}\right) \alpha_{1}=2 \chi \alpha_{0} \alpha_{2}^{*}+\sqrt{2 \gamma} \alpha_{1}^{\text {in }}+\sqrt{2 \mu} \beta_{1}^{\text {in }} \\
\tau \dot{\alpha}_{2}+\left(\gamma^{\prime}+i \varphi_{2}\right) \alpha_{2}=2 \chi \alpha_{0} \alpha_{1}^{*}+\sqrt{2 \gamma} \alpha_{2}^{\text {in }}+\sqrt{2 \mu} \beta_{2}^{\text {in }}
\end{array}\right.
$$




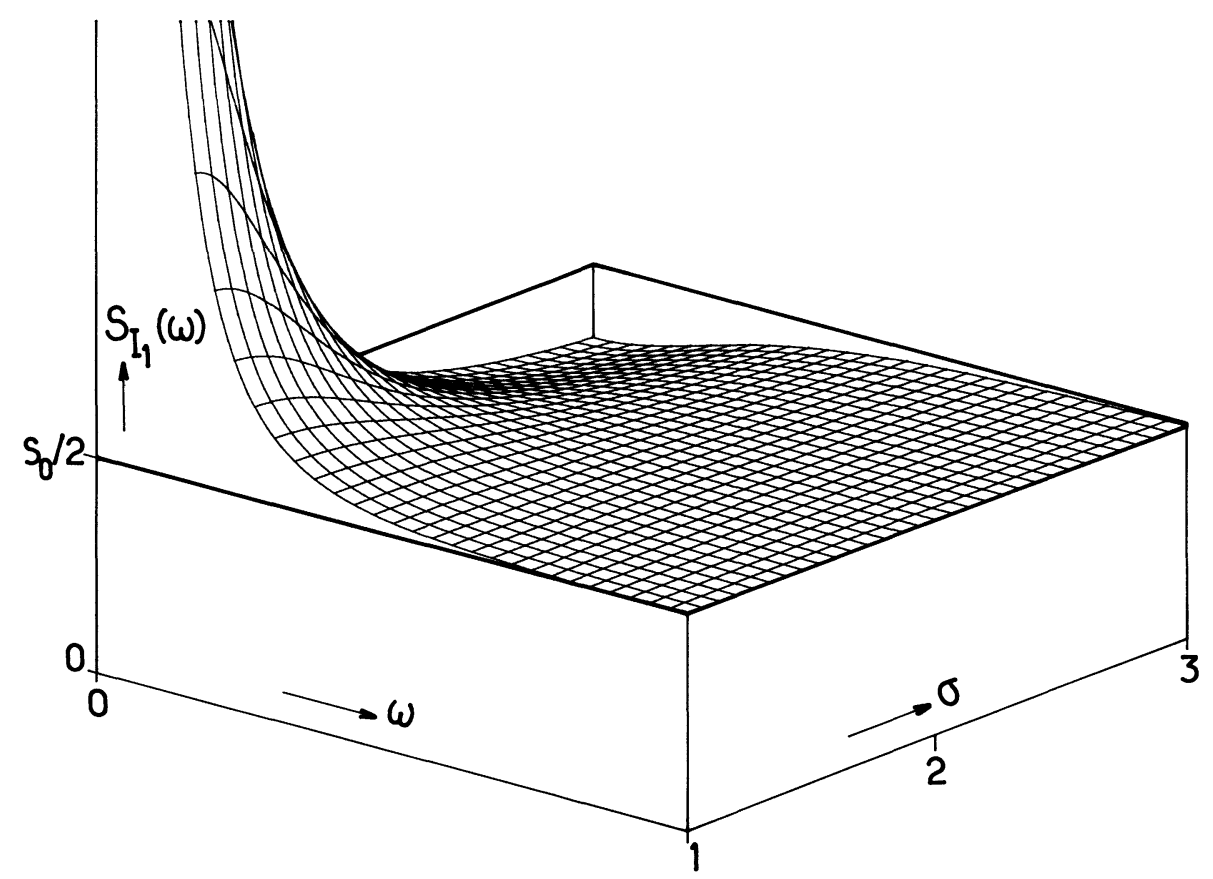

Fig. 4. - Noise spectrum $S_{I_{1}}(\omega)$ on the intensity of one signal beam as a function of the pump parameter $\sigma . S_{0} / 2$ corresponds to the standard quantum noise. $\left(\mu=0.1 \gamma^{\prime}, 0<\omega \tau<6 \gamma^{\prime}\right.$, $1<\sigma<3$ ).

The stationary values are then given by :

$$
\left\{\begin{array}{l}
\left(\gamma^{\prime}+i \varphi_{1}\right) \bar{\alpha}_{1}=2 \chi \bar{\alpha}_{0} \bar{\alpha}_{2}^{*} \\
\left(\gamma^{\prime}+i \varphi_{2}\right) \bar{\alpha}_{2}=2 \chi \bar{\alpha}_{0} \bar{\alpha}_{1}^{*}
\end{array}\right.
$$

which has a non-zero solution only when :

$$
\left\{\begin{array}{l}
\varphi_{1}=\varphi_{2}=\varphi \\
\gamma^{\prime 2}+\varphi^{2}=4 \chi^{2}\left|\bar{\alpha}_{0}\right|^{2}
\end{array}\right.
$$

A convenient choice of phase for the pump field corresponds to :

$$
\bar{\alpha}_{0}=\frac{1}{2 \chi}\left(\gamma^{\prime}+i \varphi\right)
$$

This allows us to choose a particular solution where the signal fields $\bar{\alpha}_{1}$ and $\bar{\alpha}_{2}$ are real and equal. The relation between the mean fields has been extensively studied in reference [24]. We have shown that novel features may appear, such as bistability, self-pulsing and chaos, when all the fields are detuned, including the pump field. In the present section, we assume that the pump field is on resonance with the cavity (which is not a very stringent condition, as we are in the bad cavity limit for the pump) : in this case, there exists a single stationary solution above some pump threshold. Linearizing the equations around such a solution, one obtains the following evolution equation for the difference $\delta \alpha=\delta \alpha_{1}-\delta \alpha_{2}$ :

$$
\tau \delta \dot{\alpha}+\left(\gamma^{\prime}+i \varphi\right)\left(\delta \alpha+\delta \alpha^{*}\right)=\sqrt{2 \gamma} \delta \alpha^{\text {in }}+\sqrt{2 \mu} \delta \beta^{\text {in }}
$$


(where $\delta \alpha^{\text {in }}=\delta \alpha_{1}^{\text {in }}-\delta \alpha_{2}^{\text {in }}, \delta \beta^{\text {in }}=\delta \beta_{1}^{\text {in }}-\delta \beta_{2}^{\text {in }}$ ). As in this case of exact tuning, the pump fluctuations do not affect the dynamics of $\delta \alpha$.

The mean signal fields being real, the amplitude fluctuations are equal to $r$ and proportional to the real part of $\delta \alpha$. Taking the real part of equation (34), one then gets :

$$
\tau \dot{r}+2 \gamma^{\prime} r=\sqrt{2 \gamma} r^{\text {in }}+\sqrt{2 \mu} r^{\text {in }}
$$

This equation does not depend on $\varphi$. As a result, the difference intensity spectrum $S_{I}(\omega)$ is given by equation (18). This shows that the quantum noise reduction on the intensity difference is a robust property : the Lorentzian dip displayed in figure (2) remains unchanged in the detuned case. Actually this is also true in presence of pump frequency jitter and of possible cavity length fluctuations [25]. This is not surprising, as the interpretation of this spectrum in terms of twin photons given in the introduction still holds, provided that the two signal fields are balanced.

\section{Spectrum of the intensity difference in the unbalanced case.}

5.1 GeNERAL RESULTS FOR THE NOISE SPECTRUM. - The general case where the transmission coefficients are unequal and the three optical waves are detuned from cavity resonance is important to consider in order to precisely evaluate the effect of the different parameters on the noise spectrum. In this case, the fluctuations on the difference between the two signal fields are no longer decoupled from the other fluctuations. Assuming that $\gamma_{0}^{\prime}$ is much larger than $\gamma_{1}^{\prime}, \gamma_{2}^{\prime}$, the adiabatic elimination of the pump field leads to :

$$
\begin{aligned}
& {\left[\gamma_{1}^{\prime}(1+i \Phi)-i \omega \tau+\gamma_{1}^{\prime} a\left(1+i \Phi_{0}\right)^{-1}\right] \delta \alpha_{1}-\gamma^{\prime}(1+i \Phi) \delta \alpha_{2}^{*}+\gamma^{\prime} a\left(1+i \Phi_{0}\right)^{-1} \delta \alpha_{2}=} \\
& \sqrt{2 \gamma_{1}} \delta \alpha_{1}^{\text {in }}+\sqrt{2 \mu_{1}} \delta \beta_{1}^{\text {in }}+\sqrt{\gamma_{1}^{\prime} a / \gamma_{0}^{\prime}}\left(1+i \Phi_{0}\right)^{-1}\left[\sqrt{2 \gamma_{0}} \delta \alpha_{0}^{\text {in }}+\sqrt{2 \mu_{0}} \delta \beta_{0}^{\text {in }}\right]
\end{aligned}
$$

and the similar equation with indices 1 and 2 interchanged. In this equation we have set:

$$
\gamma^{\prime}=\sqrt{\gamma_{1}^{\prime} \gamma_{2}^{\prime}}, \quad a=4 \chi^{2} \frac{\gamma_{1}^{\prime} \bar{\alpha}_{1}^{2}}{\gamma^{\prime 2} \gamma_{0}^{\prime}}=4 \chi^{2} \frac{\gamma_{2}^{\prime} \bar{\alpha}_{2}^{2}}{\gamma^{\prime 2} \gamma_{0}^{\prime}}
$$

The parameters $\Phi_{0}$ and $\Phi$ are related to the pump and signal field detunings $\varphi_{0}, \varphi_{1}$ and $\varphi_{2}$ by :

$$
\Phi_{0}=\varphi_{0} / \gamma_{0}^{\prime}, \quad \Phi=\varphi_{1} / \gamma_{1}^{\prime}=\varphi_{2} / \gamma_{2}^{\prime}
$$

Following the method exposed in the previous sections [26], one obtains a general expression for $S_{I}(\omega)$ :

$$
S_{I}(\omega)=1+\frac{N}{D}
$$

where

$$
\begin{aligned}
N & =8 \gamma_{1}^{\prime} \gamma_{2}^{\prime}\left\{\left[\omega^{2} \tau^{2}+(1+b)^{2}\left(\gamma_{1}^{\prime}+\gamma_{2}^{\prime}\right)^{2}\right]\left[g_{\mathrm{d}}^{2}-b^{2} g_{\mathrm{s}}^{2}-\omega^{2} \tau^{2} \gamma_{1} \gamma_{2}\right]\right. \\
& +\Phi\left(\Phi-b \Phi_{0}\right)\left[\omega^{2} \tau^{2}(1+2 b)\left(\gamma_{1}-\gamma_{2}\right)\left(\gamma_{1} \gamma_{2}^{\prime 2}-\gamma_{2} \gamma_{1}^{\prime 2}\right)\right. \\
& \left.+g_{\mathrm{d}}^{2}\left(\gamma_{1}^{\prime}-\gamma_{2}^{\prime}\right)^{2}+(1+2 b)\left(\gamma_{1}^{\prime}+\gamma_{2}^{\prime}\right)^{2} g_{\mathrm{d}}^{2}\right] \\
& +b \Phi_{0}\left(b \Phi_{0}-\Phi\right)\left[\omega^{2} \tau^{2}\left(g_{\mathrm{s}}^{2}+\gamma_{1} \dot{\gamma}_{2}\left(\gamma_{1}^{\prime}+\gamma_{2}^{\prime}\right)^{2}\right)-2 b(1+b) g_{\mathrm{s}}^{2}\left(\gamma_{1}^{\prime}+\gamma_{2}^{\prime}\right)^{2}-4 \gamma_{1}^{\prime} \gamma_{2}^{\prime} g_{\mathrm{d}}^{2}\right] \\
& \left.+\left(\Phi-b \Phi_{0}\right)^{2}\left[\left(\gamma_{1}^{\prime}-\gamma_{2}^{\prime}\right)^{2} g_{\mathrm{d}}^{2} \Phi^{2}-\left(\gamma_{1}^{\prime}+\gamma_{2}^{\prime}\right)^{2} g_{\mathrm{s}}^{2} \Phi_{0}^{2} b^{2}\right]\right\}
\end{aligned}
$$




$$
\begin{aligned}
D & =g_{\mathrm{s}}\left\{\omega ^ { 2 } \tau ^ { 2 } \left[\omega^{2} \tau^{2}-4 b \gamma_{1}^{\prime} \gamma_{2}^{\prime}-\left(\gamma_{1}^{\prime}+\gamma_{2}^{\prime}\right)^{2} \times\right.\right. \\
& \left.\times\left[(1+b)^{2}+\left(\Phi-b \Phi_{0}\right)^{2}\right]+4 \gamma_{1}^{\prime} \gamma_{2}^{\prime} \Phi\left(\Phi-b \Phi_{0}\right)\right]^{2} \\
& \left.+4\left(\gamma_{1}^{\prime}+\gamma_{2}^{\prime}\right)^{2}\left[\left(\omega^{2} \tau^{2}-2 b \gamma_{1}^{\prime} \gamma_{2}^{\prime}\right)(1+b)+2 \gamma_{1}^{\prime} \gamma_{2}^{\prime} b \Phi_{0}\left(\Phi-b \Phi_{0}\right)\right]^{2}\right\}
\end{aligned}
$$

where

$$
b=a /\left(1+\Phi_{0}^{2}\right), \quad g_{\mathrm{s}}=\gamma_{1} \gamma_{2}^{\prime}+\gamma_{2} \gamma_{1}^{\prime}, \quad g_{\mathrm{d}}=\gamma_{1} \gamma_{2}^{\prime}-\gamma_{2} \gamma_{1}^{\prime}
$$

The expression (39) of $S_{I}(\omega)$ has been obtained assuming that the pump beam was shot noise limited at any frequency. In order to take into account the pump excess noise, we can define two excess noise parameters $E(\omega)$ and $E^{\prime}(\omega)$ by :

$$
\left.\begin{array}{l}
\left\langle\left|\delta p_{2}^{\text {in }}(\omega)\right|^{2}\right\rangle=1+E(\omega) \\
\left\langle\left|\delta q_{2}^{\text {in }}(\omega)\right|\right\rangle=1+E(\omega)+E^{\prime}(\omega)
\end{array}\right\}
$$

where $p_{0}$ and $q_{0}$ refer to the amplitude and phase quadratures of the pump field respectively. Then, new contributions must be added to the numerator $N$ in the expression (39a) of $S_{I}(\omega)$ :

$$
\begin{aligned}
& N_{E}=E(\omega) \times \\
& \quad \times 4 b \gamma_{1}^{\prime} \gamma_{2}^{\prime}\left[\omega^{2} \tau^{2}\left(\gamma_{1}-\gamma_{2}\right)^{2}+4 g_{\mathrm{d}}^{2}\right]\left\{\omega^{2} \tau^{2}+\left(\gamma_{1}^{\prime}+\gamma_{2}^{\prime}\right)^{2}\left[(1+b)^{2}+\left(\Phi-b \Phi_{0}\right)^{2}\right]\right\} \\
& N_{E}^{\prime}=\omega^{2} \tau^{2} E^{\prime}(\omega) \times \\
& \quad \times 8 b \gamma_{1}^{\prime} \gamma_{2}^{\prime}\left(\Phi-b \Phi_{0}\right)^{2}\left[\omega^{2} \tau^{2}\left(\gamma_{1}-\gamma_{2}\right)^{2}+4 g_{\mathrm{d}}^{2}\right]\left[(1+b)^{2}+\left(\Phi-b \Phi_{0}\right)^{2}\right]^{-1}
\end{aligned}
$$

In the following we will take as an example a Gaussian frequency distribution of the amplitude excess noise $E(\omega)$ :

$$
E(\omega)=A \exp \left(-\omega^{2} / 2 \omega_{a}^{2}\right)
$$

A laser has also extra phase fluctuations due to a random phase diffusion process (responsible, in the ideal case of the Schawlow-Townes laser linewidth) of the form :

$$
E^{\prime}(\omega)=\frac{\omega_{\mathrm{p}}^{2}}{\omega^{2}}
$$

where $\omega_{\mathrm{p}}$ is on the order of the pump cavity bandwidth. Note that $N_{E}^{\prime}$ is proportional to $\omega^{2} E^{\prime}(\omega)$ which is the excess frequency noise of the pump field, and does not diverge at zero frequency.

We are now able to consider the influence of the different physical parameters on $S_{I}(\omega)$ successively.

5.2 SPECTRA IN THE RESONANT CASE. - Let us first assume exact resonance for the three fields $\left(\Phi=\Phi_{0}=0\right)$. Figure 5 displays the spectrum $S_{I}(\omega)$ as a function of the pump parameter $\sigma$ for a choice of loss and transmission parameters which are close to the experimental conditions of reference [17], i.e. with $\mu_{1} \approx \mu_{2}$ and $\gamma_{1} \approx \gamma_{2}$, so that there is a $15 \%$ difference between the mean intensities $\bar{I}_{1}$ and $\bar{I}_{2}$. Figure 5a corresponds to a pump beam at shot noise (Eqs. 39). One sees that $S_{I}(\omega)$ is above shot noise $S_{0}$ only in a very small area of the parameter space $(\omega, \sigma)$ : low noise frequencies and pump power close to threshold. This feature is easy to understand : because of unbalance, $S_{I}(\omega)$ is now slightly 


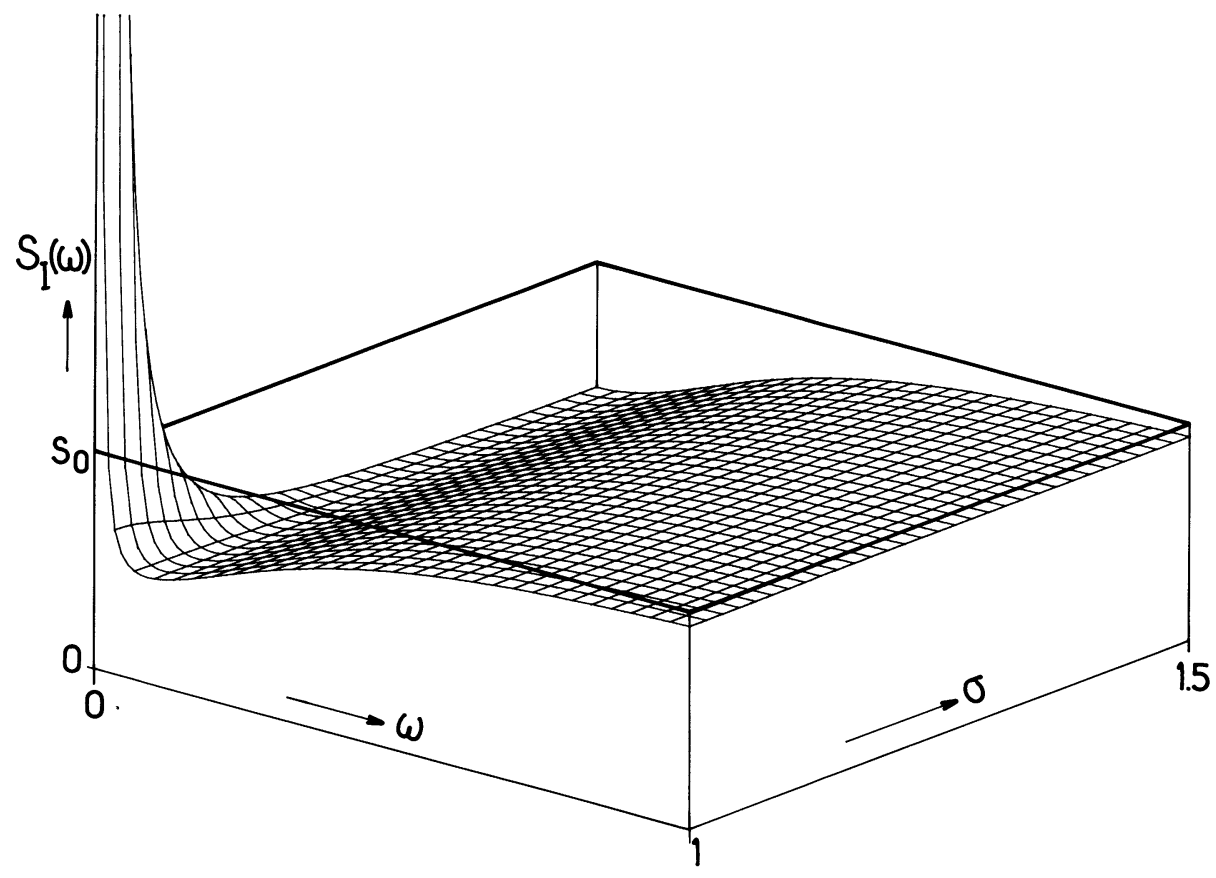

a)

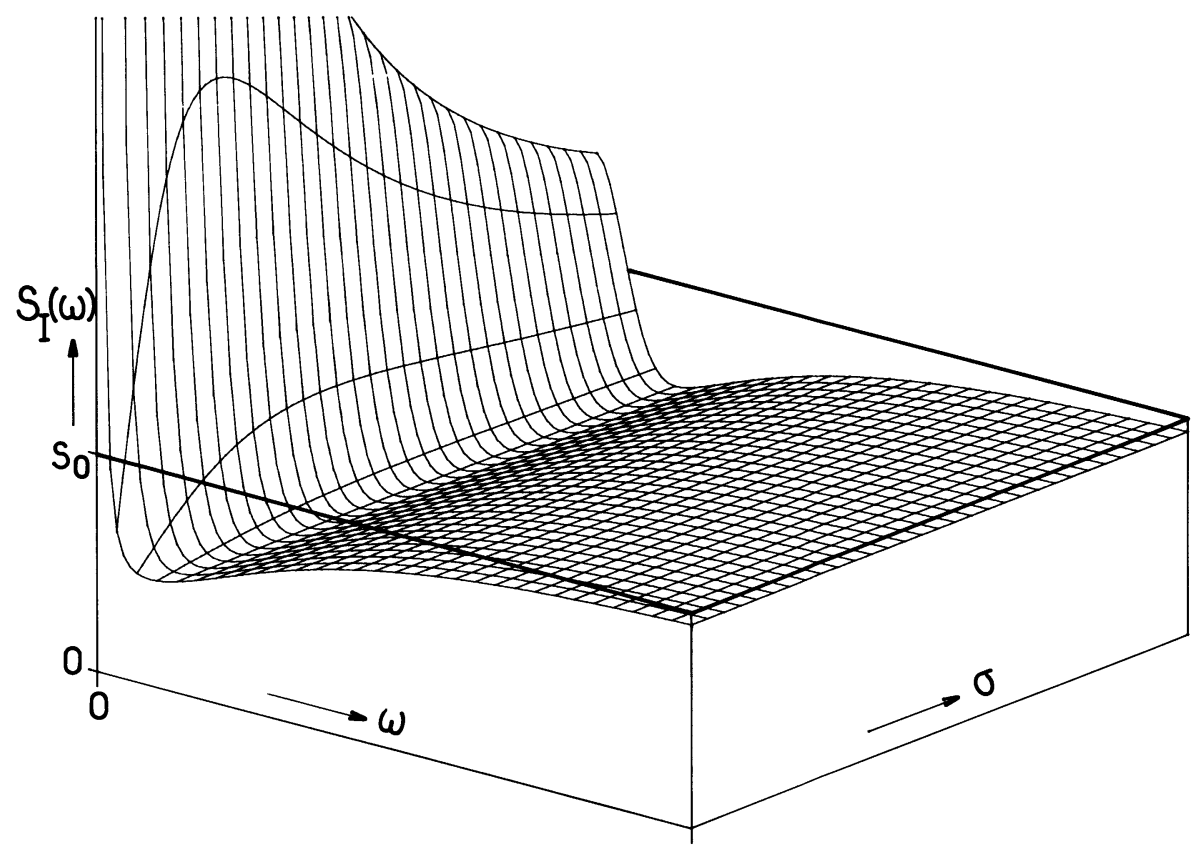

b)

Fig. 5. - Noise spectrum $S_{I}(\omega)$ on the intensity difference as a function of the pump parameter $\sigma$ in the unbalanced case. $S_{0}$ is the standard quantum noise $\left(\gamma_{1}=0.65 \gamma^{\prime}, \gamma_{2}=0.53 \gamma^{\prime}, \mu_{1}=0.38 \gamma^{\prime}\right.$, $\left.\mu_{2}=0.44 \gamma^{\prime}, 0<\omega \tau<6 \gamma^{\prime}, 1<\sigma<1.5\right)$; a) the pump beam is shot noise limited, b) the pump beam has excess amplitude noise $\left(A=100, \omega_{a}=0.3 \gamma^{\prime} / \tau\right)$. 
sensitive to the noise existing on each channel $I_{1}$ and $I_{2}$, which is significant only for $\omega \approx 0$ and $\sigma \approx 1$ (see Fig. 4). But there is still a large noise reduction factor in the major part of the parameter space.

As it appears in equations (42), (43), the second important consequence of the unbalance is to couple the pump noise $\delta \alpha_{0}^{\text {in }}$ back into $S_{I}(\omega)$. Moreover, $S_{I}(\omega)$ is only sensitive to the excess amplitude noise $E(\omega)$ of the pump. Figure 5b shows the modification of the noise spectrum in presence of an excess noise described by equation (44). One finds a large noise peak at $\omega=0$ for any value of the pump field. For higher frequencies, $S_{I}(\omega)$ goes back to the value it had without excess pump noise. Such a behaviour for $S_{I}(\omega)$ is found in the experimental spectra for $I_{1}-I_{2}[17,18]$. Of course, if the low frequency pump intensity fluctuations are too large, our simple linearized approach for the fluctuation dynamics breaks down. But the general conclusion that $S_{I}(\omega)$ is increased because of unbalance only in a limited range of low frequencies remains certainly valid.

5.3 EFFECT OF SIGNAL FIELD DETUNING. - We take a non-zero value for the signal field detuning $\Phi$, keeping the pump field detuning $\Phi_{0}$ equal to 0 . Figure 6 gives the spectrum $S_{I}(\omega)$ for a given pump parameter when $\Phi$ is varied between 0 (maximum output intensity) and $\Phi_{m}$ (OPO switch-off). One observes a large amount of excess noise for low frequencies and large detunings. In this domain, the noise spectrum has a similar shape as in the resonant case for pump values close to threshold (Fig. 5).

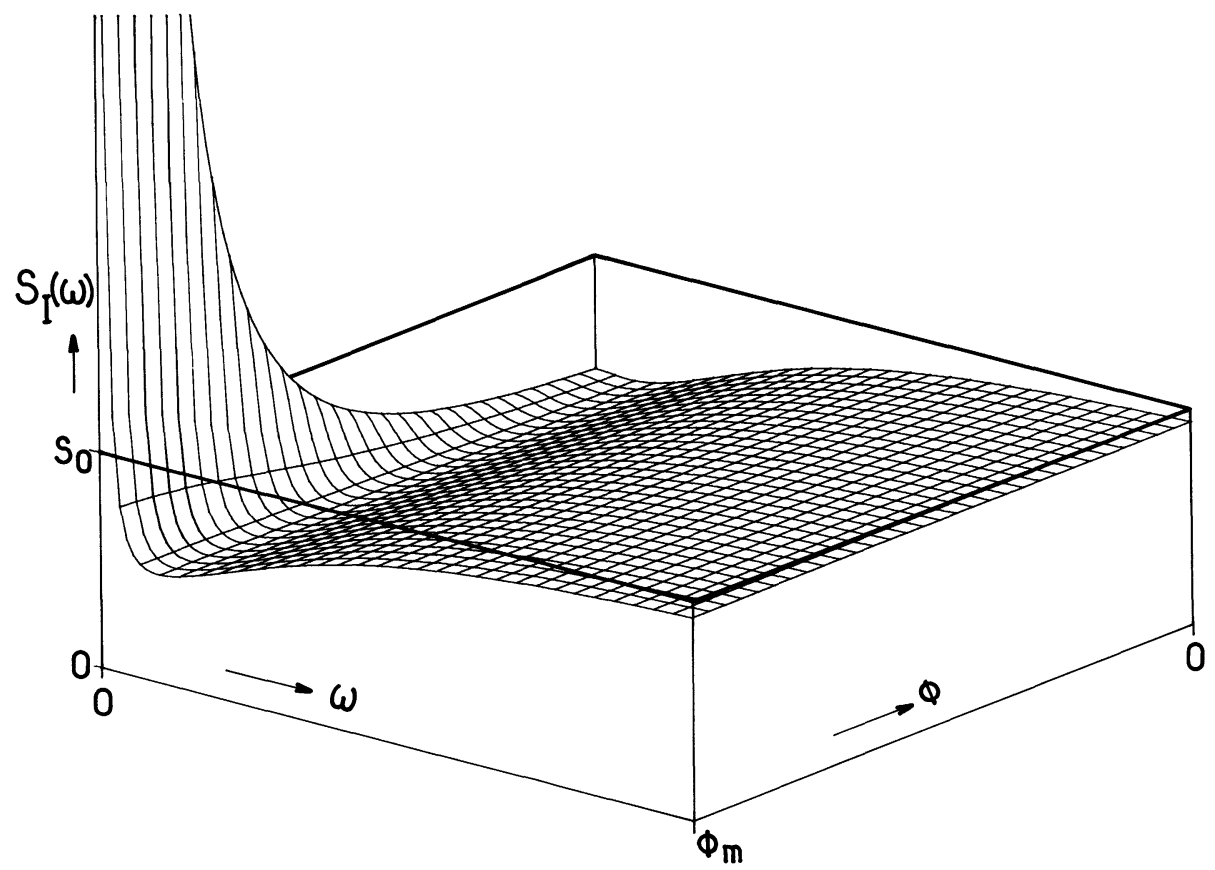

Fig. 6. - Noise spectrum $S_{I}(\omega)$ on the intensity difference as a function of the signal field detuning $\Phi$. $S_{0}$ is the standard quantum noise. $\Phi=\Phi_{\mathrm{m}}$ corresponds to the OPO switch-off, $\Phi=0$ to the maximum output intensity (same parameters as in figure 5 except $\sigma=1.2, \Phi_{0}=0$ ).

As another consequence of detuning, $S_{I}(\omega)$ is coupled to the excess frequency noise $\omega^{2} E^{\prime}(\omega)$ and in particular, to the Schawlow-Townes fluctuations of the pump laser equation 
(45). One finds that such a frequency noise has a very weak influence on the spectrum in the whole parameter space.

5.4 EFFECT OF PUMP FIELD DETUNING. - We now allow for a non zero pump field detuning $\Phi_{0}$. For a pump power close to threshold, one finds that, in the low frequency range, the detuning $\Phi_{0}$ improves the noise reduction effect by a small amount. But, on the whole, one can say that in the experimental conditions of reference [17], the pump field detuning has only a small effect on the noise spectra.

The situation is completely different at very high pump power levels (namely $\sigma>2$ ). Let us focus our attention in the parameter region where there exists a large excess noise on $I_{1}-I_{2}$ at low frequency on figure 6 , corresponding to $\Phi \approx \Phi_{\mathrm{m}}$. Figure 7 gives the variation of $S_{I}(\omega)$ as a function of $\Phi_{0}$ for $\sigma=2.5$ and keeping $\Phi \approx \Phi_{\mathrm{m}}$ [27]. One observes that the low frequency excess noise is completely suppressed for some range of $\Phi_{0}$ values. This feature is related to the occurrence of a bistable regime in the same region of the parameter space [24]. This region looks especially interesting for potential noise reduction and will be studied in more detail in a forthcoming paper.

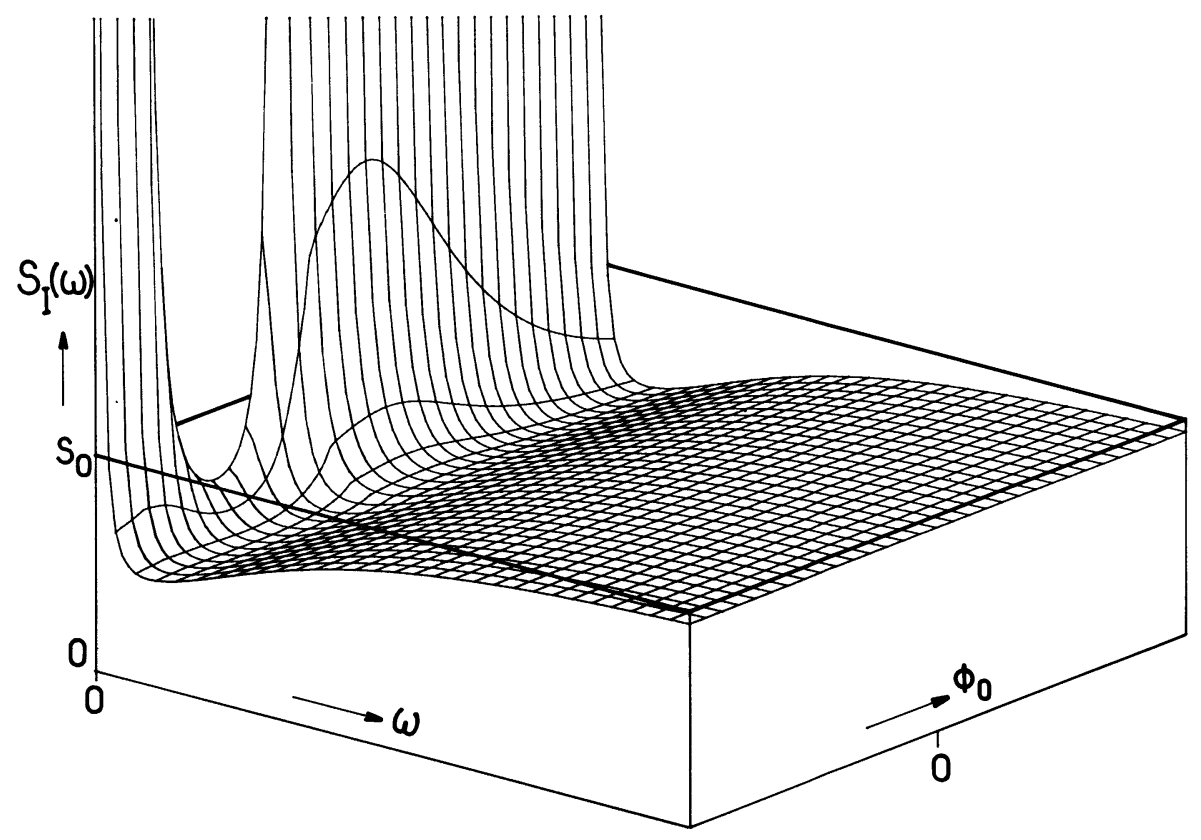

Fig. 7. - Noise spectrum $S_{I}(\omega)$ on the intensity difference close to threshold as a function of the pump field detuning $\Phi_{0} . S_{0}$ is the standard quantum noise. $\Phi_{0}$ varies between $-0.5 \gamma^{\prime}$ and $0.5 \gamma^{\prime}$ while $\Phi$ is kept equal to $0.999 \Phi_{\mathrm{m}}$. (Same parameters as in Fig. 5 except $\sigma=2.5$ ).

\section{Effect of an external balancing.}

There is a simple way to get rid of the unbalance between the mean intensities $\bar{I}_{1}$ and $\bar{I}_{2}$ : it is to insert on the more intense beam (for exemple $I_{1}$ ) a partly reflecting plate of amplitude transmission $t_{1}$ so that :

$$
\left|t_{1}\right|^{2} \bar{I}_{1}=\bar{I}_{2}
$$


Such a technique has of course a drawback : it couples back to the monitored channels the vacuum fluctuations $\alpha^{\mathrm{vac}}$ coming from the unused port of the reflecting plate. More precisely, the field $\alpha_{1}^{t}$ after the plate, impinging on the photodetectors is given by :

$$
\alpha_{1}^{t}=t_{1} \alpha_{1}^{\text {out }}+\sqrt{1-t_{1}^{2}} \alpha^{\text {vac }}
$$

The resulting monitored noise spectrum of $I_{1}-I_{2}$ is a trade-off between the low frequency noise reduction due to the cancellation of the additional noise sources and the broadband noise increase due to the coupling with extra vacuum fluctuations. Figure 8 shows $S_{I}(\omega)$ in the same conditions as Figure $5 \mathrm{~b}$ for the OPO, when the partly reflecting plate has been added on the beam (1). One observes a drastic improvement in the noise spectrum, due to the fact that the pump noise fluctuations are exactly cancelled by such a technique for $\omega=0$, and strongly reduced for all other frequencies. Also the noise peak coming from the fluctuations of $I_{1}$ is somewhat reduced. Because $t_{1} \approx 1$, there is only a very small increase of $S_{I}(\omega)$ due to $\alpha^{\text {vac }}$.

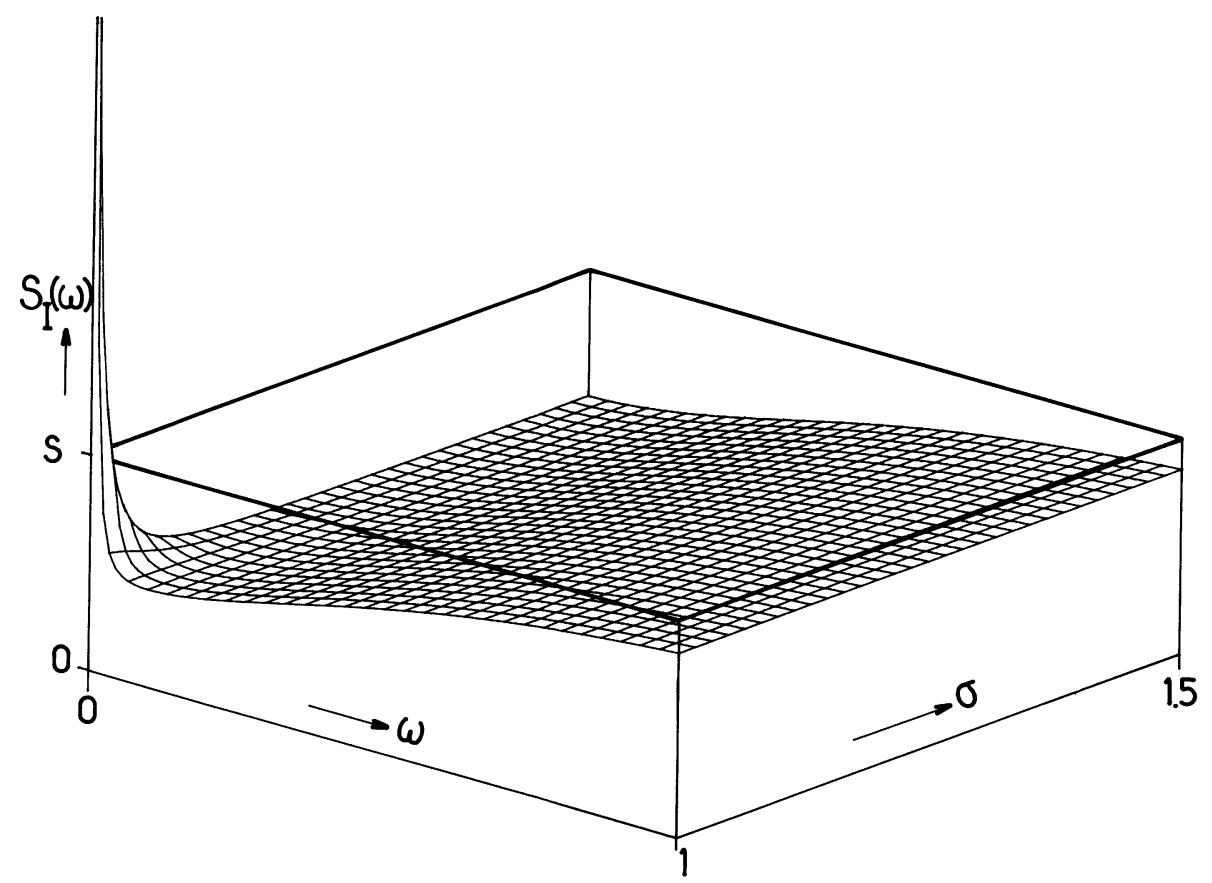

Fig. 8. - Noise spectrum $S_{I}(\omega)$, on the intensity difference as a function of the pump parameter $\sigma$ after external rebalancing so that the mean monitored intensities are equal. (Same parameters as in Fig. 5 and $\left.\left|t_{1}\right|^{2}=0.868\right)$.

One thus gets a paradoxical conclusion : losses added to a given optical system may result in a large quantum noise reduction, provided that such losses are carefully adjusted. A similar conclusion has been drawn in the case of the imperfect Michelson interferometer using squeezed vacuum [28], and in the case of intensity quantum noise reduction using passive bistable devices [29]. 


\section{Conclusion.}

In this paper, we have reviewed in detail the noise properties of the non-degenerate above threshold OPO, with the help of a semi-classical approach. The most important features which have been brought out are the following:

- the noise reduction properties of the OPO on $I_{1}-I_{2}$ are largely insensitive to disturbing physical parameters : pump power, detunings, excess noise... provided that the system is balanced with respect to its two output twin beams;

- in the case of slight unbalance, there is a noise peak on $I_{1}-I_{2}$ at low frequencies for values of the pump intensity and detunings leading to very weak output intensities ;

- it is possible to almost cancel this noise peak by a simple external balancing.

This work has been supported by a D.R.E.T. contract $N^{\circ} 87 / 091$.

\section{References}

[1] Louisell W. H., Yariv A., Siegmann A., Phys. Rev. 124 (1961) 1646.

[2] Takahasi H., Adv. Com. Systems 1 (1965) 227.

[3] Mollow B., Glauber R., Phys. Rev. 160 (1967) 1076 and 1097.

[4] Mollow B., Phys. Rev. A 8 (1973) 2684.

[5] Stoler D., Phys. Rev. Lett. 33 (1974) 1397.

[6] Graham R., Phys. Rev. Lett. 52 (1984) 117.

[7] Milburn G., Walls D., Opt. Commun. 39 (1981) 401.

[8] Lugiato L., Strini G., Opt. Commun. 41 (1982) 67.

[9] Yurke B., Phys. Rev. A 29 (1984) 408.

[10] Collett M., Gardiner C., Phys. Rev. A 30 (1984) 1386 and 31 (1985) 3761.

[11] Gardiner C., Savage C., Opt. Commun. 50 (1984) 173.

[12] Wu L., Kimble H., Hall J., Wu H., Phys. Rev. Lett. 57 (1986) 2520.

[13] Fabre C., Giacobino E., Reynaud S., Debuisschert T., SPIE 701, 1986 ECOOSA (Florence) Eds. S. Sottini, S. Trigari p. 488.

[14] Reynaud S., Fabre C., Giacobino E., J. Opt. Soc. Am. B 4 (1987) 1520.

[15] BJÖrk G., Yamamoto Y., Phys. Rev. A 37 (1988) 125.

[16] Lane A., Reid M., Walls D., Phys. Rev. A 38 (1988) 788.

[17] Heidmann A., Horowicz R., Reynaud S., Giacobino E., Fabre C., Camy G., Phys. Rev. Lett. 59 (1987) 2555.

[18] Fabre C., Giacobino E., Reynaud S., Debuisschert T., Heidmann A., Hilico L., Atomic Physics 11 (Paris 1988), Eds. S. Haroche, J.-C. Gay, G. Grynberg (World Scientific, Singapour).

[19] ReYnaud S., Europhys. Lett. 4 (1987) 427.

[20] Reynaud S., Heidmann A., to be published in Opt. Commun. (1989).

[21] Sмiтh R. G., Optical Parametric Oscillators in Laser Handbook I, Eds. F. Arecchi, E. SchulzDubois (North Holland Amsterdam) 1973.

[22] Sargent M., Scully M., Lamb W., Laser Physics, Addison-Wesley (Reading U.S.A.) 1974.

[23] Yamamoto Y., Machida S., Nilsson O., Phys. Rev. A 31 (1986) 4025.

[24] Lugiato L., Oldano C., Fabre C., Giacobino E., Horowicz R., Nuovo Cimento D, Nuovo Cimento 10D (1988) 959.

[25] The detunings $\varphi_{1}$ and $\varphi_{2}$ which appear in equations (30) as parameters may fluctuate.

The basic parameters are the pump frequency and cavity length. Any fluctuations of these 
parameters give rise to fluctuations of the detunings $\varphi_{1}$ and $\varphi_{2}$ through the OPO operating conditions. These new terms enter equation (34) as imaginary contributions. Therefore they do not appear in equation (35) and they do not affect the difference intensity spectrum.

[26] The derivation has been performed with the help of muMATH symbolic algebra package.

[27] In the actual experiment, the field detuning $\Phi$ is varied by scanning the cavity length. As the pump resonance curve is much broader than the signal resonance curve, we consider that the normalized pump detuning $\Phi_{0}$ remains constant while $\Phi$ varies.

[28] Gea-Banacloche J., Leuchs G., J. Opt. Soc. Am. B 4 (1987) 1667.

[29] Reynaud S., Fabre C., Giacobino E., Heidmann A., submitted to Phys. Rev. A. 